\title{
Empirical Research on Systems Thinking and Practice in the Engineering Enterprise
}

\author{
Donna H. Rhodes, Caroline T. Lamb and Deborah J. Nightingale \\ Massachusetts Institute of Technology \\ Building NE20-388, 77 Massachusetts Avenue \\ Cambridge, MA 02139 \\ http://seari.mit.edu
}

\begin{abstract}
The paper discusses recent and ongoing research on engineering systems thinking and practices within the Engineering Systems Division at the Massachusetts Institute of Technology. . The research seeks to impact the effectiveness of systems engineering in modern enterprises through development of new empirical-based knowledge related to systems thinking and practice in engineering. The paper will discuss research progress and outcomes to date as they apply to improving the effectiveness of systems engineering practice and competency development in industry, government and academia. The research involves highly collaborative engagement, use of grounded theory methods, and both quantitative and qualitative analysis. The challenges and lessons learned in performing research of this nature and applying non-traditional methods in systems engineering research are discussed.
\end{abstract}

Keywords - systems thinking, collaborative distributed systems engineering, collaborative systems thinking, enterprise thinking

\section{INTRODUCTION}

The practice of systems engineering has advanced significantly over the past decade in response to new challenges. As a result, the demand for systems engineering practitioners has increased, yet at the same time the engineering workforce is declining in the US and other countries [1]. Studies also cite an erosion of engineering competency, particularly in government and the aerospace/defense industry [2]. The development of systems competency is critical given the challenges faced; yet, the systems community lacks the empirical basis for developing a well-informed, data driven strategy for addressing this need. At the same time, society as a whole is faced with increasing complex systems problems in critical infrastructure, energy, transportation, communications, defense, and others areas. The increasing demand for systems leaders coupled with the growing need to address significant socio-technical challenges [3] motivates research in engineering systems thinking and practice.

This area of research requires empirical studies and casebased research for the purpose of understanding how to achieve more effective systems engineering practice through understanding of the context in which systems engineering is performed and the factors underlying the competency of the systems workforce. The paper describes three ongoing research thrusts with highlights of interim research outcomes. The first research effort is focused on building empirical knowledge of the enablers, barriers and precursors of the development of systems thinking in individual engineers, and thus far has included a study within the aerospace industry [4], and mini-study in an aerospace government agency. A second line of research is looking at effective socio-technical practices of collaborative distributed systems engineering [5]. A third research project $[6,7]$ seeks to develop an empirical basis for collaborative systems thinking, defined as "an emergent behavior of teams resulting from the interactions of team members and utilizing a variety of thinking styles, design processes, tools, and languages to consider system attributes, interrelationships, context and dynamics towards executing systems design". The project examines collaborative systems thinking through examining the alignment of enterprise culture and standard processes.

The nature of these research topics does not permit an easy fit into the traditional structure of academic institutions and funding agencies. An in-depth understanding of engineering practice is necessary, and at the same time, an orientation in social science is also needed. The exploratory nature of this early research is not well suited to a method that formulates and tests hypotheses, as initial knowledge must be gathered and synthesized in order to form meaningful hypotheses. Thus, the use of ground theory has proved to be important for this early stage of developing the research. Whereas traditional deductive research starts with a hypothesis and then seeks evidence to prove or disprove the hypothesis, exploratory research starts with an interesting question or area of inquiry and ends with a set of hypotheses that form the basis for new theory, known as grounded theory research. Grounded theory research is characterized by concurrent and systematic data collection, analysis, and theory development $[8,9]$. Because grounded theory research utilizes a systematic process to collect and analyze data, it leads to a more accurate process of discovery. Case studies are an important part of the research approach, as these are well suited for exploratory research because they are a flexible and effective means to gather many types of information, and helpful in establishing external validity of the data collected as well as increasing the generality of findings [10]. Effective use of empirical research methods is a necessary and challenging factor [11]. In light of the challenging situation of undertaking academic research on this subject, the authors have found that having an appropriate landscape for the research program has been essential. 


\section{RESEARCH LANDSCAPE}

The nature of the academic research environment has a critical impact on the formulation of research programs and the resulting research outcomes, and the specific academic unit within which research is undertaken implies a unique intellectual climate and topography. A research landscape is the overall mental model under which research is formulated, performed, and transitioned to practice. It provides the context for the research agenda, research methods, and specific projects, and determines the community of interest on which research should have impact. The research landscape both opens possibilities for and also constrains funding sources and sponsors, which can have significant influence on research outcomes and transition to practice.

The MIT Engineering Systems Division (ESD) is a new kind of interdisciplinary academic unit that spans most departments within the School of Engineering, as well as the School of Science, School of Humanities, Arts, and Social Sciences, and the Sloan School of Management. This setting offers a robust research and learning environment for advancing engineering practice to meet the contemporary challenges of complex socio-technical systems in an academic field of study MIT calls engineering systems.

Engineering Systems is a field of study taking an integrative holistic view of large-scale, complex, technologically-enabled systems with significant enterprise level interactions and socio-technical interfaces, encompassing and also extending the footprint of systems engineering [3]. Foundational papers ${ }^{1}$ provide an elaborated definition and description, along with additional discussions of research within this new field. Research in this field is highly interdisciplinary in nature, engaging faculty and students from a broad base of disciplines and domains. Research projects in engineering systems use both quantitative and qualitative approaches, and typically involve deep engagement with real world industry and government projects.

The field of engineering systems as a meta-field for systems engineering [3] is viewed by the authors as a highly appropriate landscape for undertaking research on systems thinking and practice in the engineering enterprise. It would be less than optimal for a social science department alone to undertake research that examines large scale technologyenabled innovative because performing case studies in these types of endeavors is difficult without engineering practitioner knowledge. Yet, the typical engineering department is not well suited for performing social science based research. As such engineering programs are created and transformed, more suitable academic venues for performing this type of research through interdisciplinary expertise and approaches will be created $^{2}$. These types of programs foster collaboration among

\footnotetext{
${ }^{1}$ Refer to MIT Engineering System Division's website for many papers: http://esd.mit.edu/WPS/wps.html

${ }^{2}$ The Council on Engineering Systems Universities (CESUN, http://www.cesun.edu ) is a body of over 50 universities collaborating on evolving this broader field of engineering.
}

engineering, management, and social scientists; and application of methods to bridge the disciplines. Rouse [12] describes why such research needs to occur in engineering settings, asserting that engineers are uniquely suited to see the whole enterprise, or systems, and tease out links that might not be seen from a management or purely social perspective. Before discussing the three areas of research that are the subject of this paper, it is useful to first examine traits of the modern engineering systems leader.

\section{TRAITS OF SYSTEMS LEADERSHIP}

Empirical research on systems thinking and practice will only have appropriate impact if it is undertaken with an eye toward the desired traits of professionals needed for modern systems. In 1962, Hall [13] identified five traits of the ideal systems engineer as: (1) an affinity for the systems point of view, (2) faculty of judgment, (3) creativity, (4) facility in human relations, and (5) a gift for expression. Certainly these traits are still fundamental for the ideal systems thinker in an engineering enterprise. Yet, as a broader understanding of complex engineering systems is developed, a new kind of professional emerges. These individuals will be powerful integrative leaders, and the engineering educational system has a significant challenge in producing such individuals to address the challenges of large-scale complex engineering systems [3]. Further, these leaders will need to consider the technological components as part of a larger engineering system (which includes the enterprise) and utilize approaches different from those based on the traditional engineering science paradigm.

Modern systems professionals must consider the context in which the system operates as a design variable rather than a constraint, and be concerned with the design of the organization that has to develop the system or product; the regulations and public policies governing its use and disposition; the marketing; and the relationship with suppliers, distributors and other participants in the value chain. As such, the design process performed by these types of systems professionals includes physical attributes that are the domain of traditional engineering; process attributes that are the domain of both engineers and managers; and context attributes that traditionally have been the domain of managers, governments, and social scientists. Academia faces the challenge of producing new societal leaders who are: (1) skilled intellectually at dealing with the many crucial technological dimensions of our society; (2) have the practical results orientation that is characteristic of engineering professionals; (3) have the courage based on early experience to take on the most difficult systems problems; and (4) have the leadership skills to bring others forward as they themselves progress [14].

Contemporary engineering leaders must be capable of situational leadership [15], possessing the ability to make decisions using either of two unique perspectives based on situational context. Products and systems are developed to 
satisfy well defined performance objectives, and in addition, these may be integrated into larger collaborative system to provide a complex capability to satisfy a broader base of stakeholders. As such, engineering leaders sometimes need to make decisions oriented toward delivering a well defined product while at other times may have responsibility (or shared responsibility) for delivering a complex solution comprised of a cooperating set of legacy systems and associated enterprises. In the latter, the goal is to address a higher order societal need or capability greater than the simple sum of the component system capabilities. Decision criteria and trades are unique depending on the perspective used, and at times, the engineering leader must be able to make decisions to sacrifice or delay specific product capability (or features) in order to realize the system family's overall capability in which they are a contributing part. This ability to move back and forth between these two perspectives, making effective decisions given situational context, is an important trait in systems thinkers for many of today's systems.

It can be concluded that engineering systems thinking and practice demands higher order abilities for analysis and synthesis given contemporary system challenges. Differences in types of systems thinkers have emerged in the early research, as well as commonalities. The traits of systems leadership that are necessary, both today and in the future, are considerations in uncovering the empirically derived knowledge the research program seeks to discover. Three research thrusts are now discussed, including implications of the research for the practice.

\section{THREE AREAS OF RESEARCH}

\section{A. Engineering Systems Thinking in Individuals}

The development of generalized systems thinking [16] has been studied empirically; however, the development of engineering systems thinking has remained largely unexplored. Ref. [17, 18] characterize engineering systems thinking as distinct from systems thinking in general. Ref. [19] describes an empirical study performed to better understand systems thinking development in engineers, and specifically how senior systems engineers develop. The research focused on three key research aspects: (1) enablers, barriers, and precursors to the development of systems thinking in engineers; (2) specifics of how senior systems engineers develop; and (3) the mechanisms that develop systems thinking in engineers. Designed as an exploratory and inductive study, this research utilized a series of interviews and surveys to gather data on the systems thinking development process in a population of over 200 engineers, primarily in the aerospace and defense industry. One research outcome was a systems thinking framework, definition and accompanying conceptual illustration. This asserted five foundational elements to descriptors in a systems thinking framework: (1) componential, (2) relational, (3) contextual, (4) dynamic, and (5) modal elements. As a result, it offers the empirically derived definition, "Systems thinking is utilizing modal elements to consider the componential, relational, contextual, and dynamic elements of the system of interest."

This study [19] found that although systems thinking definitions diverge, there is convergence on mechanisms that enable and obstruct systems thinking development. Data based on subjects in industry shows primary mechanisms enabling systems thinking development to include: (1) experiential learning, (2) various individual characteristics, and (3) a supporting environment. MIT researchers conducted a smaller follow-on study of systems engineers in a government agency in the space sector. While having too few subjects to show statistical significance, this study did provide indications that the same three factors were important [20].

Implications for Systems Engineering Practice. Ref. [19] used the results of interviews with systems engineering practitioners and expert panelists to derive a set of general recommendations for government, industry, and academia. Examples of recommendations for government included adjustment of policies to emphasize experiential learning for systems thinking development; changes in acquisition strategy to provide more programs and opportunities for engineers to develop systems thinking; and promoting systems thinking. The recommendations of the study for industry include structuring systems thinking interventions to emphasize experiential learning; offering systems programs to teach systems skills and systems thinking; filtering and fostering identified individual characteristics in systems organizations; and providing an environment supportive to the development of systems thinking. For academia, the study recommends programs to teach systems skills and systems thinking; structuring programs and courses to emphasize experiential learning; and continuing research on mechanisms for effective systems thinking.

Extensive literature review and two studies on engineering systems thinking in industry [19] and government [20] provide empircal data on engineering systems thinking stategies and interventions. These studies inform initial recommendations for improving the education of systems engineers in academia and in corporate/government education programs. Three empirically derived recommendations are:

1. Educate engineers to think more deeply about systems in their context or environment. This should include improving the ability to understand system boundaries, and how these may shift over time. Engineers need to be educated to understand how systems react to internal/external impacts. This includes developing knowledge of constructs for impact analysis and methods decision making.

2. Develop 'situational leadership' abilities of engineers [15] in regard to how to make decisions from multiple levels perspectives and levels - component, system, system of systems. This style of leadership necessitates an 
improved understanding of the decisional trade-off process for local versus global system value delivery.

3. Provide more classroom and experiential learning opportunities in regard to the temporal context of systems. This includes how to think about systems and system interactions within and across life cycle phases, and natural value-centric time scales [21]. Included is the critical ability to anticipate and model future scenarios, and how system decisions in present time may enable flexibility for the future.

In addition to informing the enhancement of competency development programs, these findings also inform the larger MIT advanced systems engineering research program in evolving methods for advancing the practice of systems engineering. ${ }^{3}$

\section{B. Collaborative Distributed Systems Engineering}

The complexity and size of many engineering programs has led to the need to distribute engineering effort across business units, corporations, and geographic boundaries. The means to collaborate in a distributed workforce involves challenges that are both technical and social in nature, and having both logistical and cultural considerations. While there has been some previous experience with distribution of work, new factors lead to a desire to further consider the factors involved in collaborative distributed engineering through empirical research. Ref. [5] describes an exploratory research study of selected US aerospace and defense companies to identify emerging best practices in collaborative distributed systems engineering (CDSE), focused on three major objectives.

The first objective focused on in this research was to define successful social and technical CDSE practices by examining companies currently performing this practice, and lessons learned. Successful CDSE involves many factors, with eleven addressed by this research study: (1) use of CDSE and collaboration tools; (2) scheduling and conduct of meetings; (3) communication; (4) training of engineers; (5) overcoming social and cultural differences; (6) making decisions; (7) adapting the product; (8) overcoming issues and barriers; (9) determining and measuring CDSE benefits; (10) managing knowledge and data; and (11) coordination of processes. A second research objective was to identify key CDSE issues encountered; barriers and how these were overcome; and practices that were tried yet failed. This new understanding can inform companies starting to perform CDSE in order to prevent them from repeating the same mistakes. The third objective was to identify topics for future research to undertake more rigorous and extensive studies toward generalizable outcomes.
The motivations for this research include the shrinking of aerospace and defense budgets; scarcity of resources and more demanding requirements; and aerospace and defense enterprises that are expected to deliver a more capable product in less time and with fewer resources. Ref [5] states:

To achieve this tough mission, the enterprises that comprise the US aerospace and defense industries must form strategic partnerships and collaborations to utilize their respective resources, knowledge, and expertise to meet their customers' needs. Collaboration, be it between competing companies or within different divisions of the same company, is necessary for the survival of each company and the defense industry. In the past, aerospace and defense company relationships consisted mostly of a prime contractor, with sub-contractors providing a specific hardware or software subsystem, as specified by the prime contractor. Today, aerospace and defense company relationships are moving more toward that of "partners" where the previous supplier or sub-contractor for hardware or software subsystems is now sharing in the overall system design and engineering efforts. Since the partner companies and intra-company divisions are still geographically distributed throughout the US, it is necessary for contractors to perform collaborative, distributed systems engineering (CDSE) over several geographical locations. Previous research has demonstrated that the design practices of distributed design teams differ from those of traditional, co-located teams. However, many companies today are performing CDSE using processes and methods developed for traditional SE environments and are therefore encountering many issues. Successful SE practices are difficult to carry-out when performed by a traditional, collocated enterprise. The addition of geographic distribution and cross-company or intra-company collaboration in SE presents a myriad of social and technological challenges that necessitate new and different SE methods for success. Best practices for CDSE are currently unknown (or undocumented).

The research investigated and captured CDSE lessons learned and success factors based on two case studies carried out at two US aerospace and defense companies. The case studies examined many pertinent factors, including collaboration scenarios; collaboration tools; knowledge and decision management; SE practices and processes; SE process improvements; SE culture; SE project management; SE organization; and SE collaboration benefits and motivation. The research has resulted in a preliminary set of CDSE "success factors" which extend from the research, as well as from factors uncovered in the literature. Five examples of the cited success factors are summarized in Table 1.

\footnotetext{
${ }^{3}$ Systems Engineering Advancement Research Initiative, SEAri, http://seari.mit.edu
} 
Table 1. Example CDSE Success Factors [5]

\begin{tabular}{|c|c|}
\hline Success Factor & Description \\
\hline Establish Trust & $\begin{array}{l}\text { Trust enables open communications between } \\
\text { team members and inspires confidence in the } \\
\text { final product and cooperation between teams. }\end{array}$ \\
\hline $\begin{array}{l}\text { Invest in } \\
\text { Up-front Planning } \\
\text { Activities }\end{array}$ & $\begin{array}{l}\text { Spending more time on the front end activities } \\
\text { and gaining team consensus shortens the } \\
\text { implementation cycle. It avoids pitfalls as } \\
\text { related to team mistrust, conflict, and mistakes } \\
\text { that surface during implementation. }\end{array}$ \\
\hline $\begin{array}{l}\text { Perform Visual } \\
\text { Management of } \\
\text { the Development } \\
\text { Process }\end{array}$ & $\begin{array}{l}\text { Visual management of the development process } \\
\text { may be useful in establishing a sense of team, } \\
\text { as well as keeping the team immediately up-to- } \\
\text { date on important programmatic and product } \\
\text { related issues. This visual management may be } \\
\text { possible by using the collaboration tools or } \\
\text { environments and/or team room displays. } \\
\text { Imagine an online collaborative environment, } \\
\text { and upon logging in, immediately being } \\
\text { informed of a subsystem's current testing or } \\
\text { development status (perhaps in red, yellow, } \\
\text { green). Or similarly, entering a CDSE team } \\
\text { room to find the color-coded schedule progress } \\
\text { of each team. These visual cues provide } \\
\text { immediate feedback without having to scour } \\
\text { schedules, requirements, or test data and are } \\
\text { relatively simple to implement. }\end{array}$ \\
\hline $\begin{array}{l}\text { Define Decision } \\
\text { Making } \\
\text { Responsibilities }\end{array}$ & $\begin{array}{l}\text { A decision making matrix outlines the roles } \\
\text { leadership plays in each of the major decisions. } \\
\text { Included in the matrix is which leader makes } \\
\text { the actual decision, which leaders need to be } \\
\text { consulted beforehand, and who should be } \\
\text { informed after decisions are made. }\end{array}$ \\
\hline $\begin{array}{l}\text { Provide CDSE } \\
\text { Training }\end{array}$ & $\begin{array}{l}\text { Training can make a huge impact, as } \\
\text { exemplified in a case of the GE 6sigma black } \\
\text { belts. GE recognized the need for virtual } \\
\text { teaming as a future key mission critical need } \\
\text { and has trained all of their "Black Belts" since } \\
1998 \text { in virtual teaming. The same type of } \\
\text { training can be used throughout SE } \\
\text { organizations to inform the CDSE work } \\
\text { environment. }\end{array}$ \\
\hline
\end{tabular}

Research suggests that success in collaborative distributed systems engineering cannot be achieved without first overcoming several possible barriers, including many of the issues encountered by co-located teams and additional ones unique to the collaborative distributed team. In addition to typical issues with time zones and misaligned schedules, Ref. [5] identifies potential barriers unique to CDSE, for example:

Too many Perspectives: Although also cited as a CDSE benefit, research has demonstrated that the diversity of knowledge held by collaborating systems engineers (or any collaborating teams with diverse experiential and intellectual backgrounds) can also be a barrier to successful knowledge sharing. It is difficult to share and understand knowledge when engineers do not share the same social, occupational or cultural background. This is because different experiential and intellectual backgrounds can lead to different "perspectives, priorities, typical approaches to problem solving, and even terminology." These differences can often be overcome when collaborators work together frequently in highly interactive settings. However, in distributed collaboration, engineers are limited in face-to-face contact and the collaboration settings are not highly interactive or very frequent.

Implications for Systems Engineering Practice. The research on collaborative distributed systems engineering identified thirteen "success themes" related to the areas of collaboration situation and management; collaboration tools; knowledge, data and decision management; SE processes and practices; and the social and cultural environment. An example of a success theme [5] is:

Program kick-off face-to-face, and regularly scheduled face-to-face meetings are necessary to build and maintain relationships and trust between teams. One project team in the study cited that issues of mistrust, company cultural differences, and misunderstandings have been remedied by repeated interactions and the ability to build relationships over time.

The success themes, while not fully validated, may provide useful considerations for enterprises faced with implementing collaborative distributed systems engineering.

Exploratory studies have uncovered differences in maturity in regard to several factors which foster or inhibit collaborative distributed systems engineering. Additional studies will permit identification of further factors, and pilot studies can provide a mechanism to validate the factors. As researchers consider the transition to practice a mechanism of interest is an assessment instrument that could aid organizations in assessing their readiness to successfully undertake collaborative distributed engineering activities, based on a collaborative maturity factor [5].

This study by nature of its sponsorship focused on cases only within the US defense industry. It should be noted that the challenges of collaborative distributed systems engineering are faced by both defense and commercial industries on a global basis, as informed by discussions the authors have had with a diverse set of of government and industry leaders. There is a need for performing many additional enterprise studies to understand the commonalities and differences to formulate the empirically derived guidance to inform the engineering practice. 


\section{Collaborative Systems Thinking}

A third area of research is investigating the enablers and barriers to team-based systems thinking, termed collaborative systems thinking [22], as examined through organizational culture and standardized engineering processes. Initial results from this research $[6,7]$ reinforce themes found in systems engineering, psychology, management, and organizational learning literature. Example themes include the harmful effects of divisive allegiances on teams, the criticality of a strong end goal, the negative impact of engineers' tendencies to work alone and complete work at the last minute, and the benefits of using multiple media to communicate design information.

Collaborative systems thinking (CST) is a construct coined to differentiate team-based systems thinking from individual systems thinking. CST, like systems thinking at the individual level, deals with managing complexity, understanding interactions and interdependencies, and handling cross-disciplinary, or multi-disciplinary knowledge - the traits of systems thinking in engineers [19]. The difference with CST is that these traits are not necessarily handled by one individual, but emerge through the interactions of a group of individuals. Group interactions are influenced by an organization's culture, team norms, a team's physical environment, and established engineering practices and processes.

Research into CST is motivated by the systems thinking skills shortage within the engineering workforce, especially within the aerospace industry. This skill shortage is further aggravated by industry demographics with greater than $25 \%$ of the workforce eligible for retirement by 2011, citing National Academy of Engineering estimates [1]. Experiential learning was identified as one of the three leading enablers of systems thinking development [19]. As experienced engineers retire, the industry will lose a disproportionate number of its systems thinkers. While individual systems thinkers are important contributors to system design, teams are the fundamental work unit in today's organizations and there has been no research into the enablers and barriers to systems thinking development within teams. These factors combine to form a need to accelerate the development of systems thinkers and find new ways to leverage the benefits of systems thinking. It is expected that insight into teams and CST will help to alleviate the problem. The objective of this research is to identify patterns of culture and process that enable teams, as a collective, to realize the benefits of systems thinking. Research into team-based systems thinking benefits from its ability to draw upon research from diverse fields and sources, including: systems engineering; systems thinking; team cognition; design thinking; psychology; process design; organizational theory; and engineering case studies.

While the initial results provide some insight into the development of CST, other questions remain unanswered. Team composition is one such question, with interviewees expressing diametrically opposed opinions: 1) that individual systems thinking is a necessary condition for CST;2) that only a systems thinking leader is required; and 3) that CST is an emergent property with no systems thinking preconditions on the team members. Additionally, the preliminary results are vague on how standard process acts as an enabler or barrier to CST beyond the consensus that they are important.

The research project is presently undertaking case studies to examine engineering teams in order to expand and validate the list of enablers and barriers to collaborative systems thinking development. Through field study, the research seeks to identify patterns of organizational culture, team norms and standard process usage as supportive for the development of collaborative systems thinking.

Implications for Systems Engineering Practice. This area of research is currently in progress and therefore implications for practice are yet to be fully considered. In this early phase where the research is informed by literature sources, adjunct research, and pilot interviews, a few generalizations about CST can be drawn that have the potential to inform practice.

1) Effective communication is a necessary condition for CST: Communication among engineers is not limited to the written and spoken word. Part of good communication in a design team is the use of sketches, drawings, mathematical equations and models. While the use of computer modeling tools may be called out in standard processes, informal sketching is very important for the creative process during early design and to help team members share ideas with one another.

2) Ability to engage in divergent and convergent thinking is an enabler for CST: Engineers excel at convergent thinking-beginning with a problem and finding a solution. Divergent thinking begins with a requirement, or need, and asks questions to explore the design space and to generate a large number of design possibilities. The challenge, as identified by interviewees, is in fostering open and critical discussion of design alternatives during the divergent phase without premature convergent thinking.

3) Product orientation is an enabler for CST: Literature on high-performing teams calls for the establishment of clear goals as an enabler to success. Likewise with CST, a clear direction and commitment to the end product is seen as an enabler for better communication, willingness to cross discipline boundaries, and ability to make tradeoffs that benefit the end system, rather than a single component or function. Interviewees recalled experiences with small companies or exciting programs as having atmospheres within which team members were able to develop CST.

4) Team awareness may be an enabler for CST: CST is about identifying and leveraging interactions, interfaces 
and cross disciplinary knowledge. Team awareness is an individual trait that indicates awareness of what other on the team are working on and also what others on the team know. This knowledge, when universally held, enables team members to preemptively share information with those who need it and better coordinate efforts toward improving a system design.

5) The 'hero' culture is a barrier to CST: Engineering culture, a recognized intra-organizational culture, is typified by the "lone engineer" working late nights to heroically finish the project. Engineering culture also fosters a tendency to procrastinate. The tendency to reward the "hero" who comes through in the end is a barrier to teamwork and to the goals of identifying and addressing concerns early in a program through team interaction and sharing of information.

6) Team segmentation is a barrier to CST: Teams may segment, or form subgroups, along functional lines, because of differences in opinions, or differences in goals. Whenever a team forms subgroups, information flow is impacted. Additionally, the ability to openly discuss and debate interactions and alternatives is hampered by allegiances to the subgroup. Functional alignment was the most commonly sighted reason for teams forming subgroups. The resulting 'turf protecting' results in missed opportunities to leverage cross-domain knowledge.

7) Culture and process are important elements in CST: Psychology, management and engineering design literature all point to culture and process as moderators on team effectiveness and the ways in which work is accomplished. Likewise, interviewees were unanimous in their belief that CST is influenced by culture and process. However, field work is required to observe and categorize specific ways in which process acts as an enabler or barrier to CST.

\section{DISCUSSION AND CONCLUSION}

The growing demand for systems professionals in complex engineering systems drives the need to grow the size of the systems engineering workforce, as well as to enhance systems thinking capacity of individuals and teams. Using ad-hoc approaches and anecdotally based assumptions will inhibit satisfying these needs. There is an urgent need for empirical studies to derive effective principles and mechanisms for ensuring systems thinking capacity of enterprises.

There are significant challenges in conducting research on engineering systems thinking and related interventions. The first challenge lies in the fact that this specific type of research is relatively new and therefore the studies are largely exploratory in nature. The scope of the studies is limited in part due to the sponsorship of research, wherein it is difficult to accomplish cross-domain studies to achieve generalizable results. The venue in which to undertake such research is also a significant factor and a challenge in itself. In the view of the authors, knowledge of engineering is critical to this research, but on the other hand, engineers do not typically have an understanding of social sciences which are also a necessary part of the research. Further, engineering academic units do not always view such research as suitable for the field. Access to subjects is another significant challenge, which can be overcome through various means such as leveraging consortia. Collaborative engagement with sponsors is an essential part of the research approach, and has strongly validated the need and importance for this overall area of research.

A number of additional research projects are underway that relate to this overall area of engineering systems thinking research, including planned extensions of the three areas that have been described previously in this paper. An ongoing joint research project between MIT and MITRE Corporation is performing a series of case studies to describe the social contexts of enterprise systems engineering. Although the cases are not yet available as of the publication of this paper; the intention is to make these publicly available in the near future. These cases will be informative in regard to the engineering systems thinking challenges and situations faced in real world programs. Another line of new research is examining the harmonization of systems engineering with enterprise contexts.

Thus far, engineering systems thinking research has largely been exploratory in nature, using approaches such as grounded theory toward a first step in obtaining preliminary findings, heuristics, and researchable hypotheses. Many further descriptive-based studies are needed to expand the dataset and to validate research outcomes toward the achievement of normative and prescriptive outcomes. Modern enterprises and society as a whole require understanding, strategies, and interventions to enable effective engineering systems thinking in order to address current and future challenges. The research projects described in this paper are examples of the foundational research that is necessary and will ultimately lead to more scientific and rigorous studies as major research programs are initiated.

\section{ACKNOWLEDGMENT}

The authors gratefully acknowledge the funding for this research provided through the Lean Advancement Initiative (LAI, http://lean.mit.edu) and the MIT Systems Engineering Advancement Research Initiative (SEAri, http://seari.mit.edu). 


\section{REFERENCES}

[1] D. Black, D. Hastings and the Committee on Meeting the Workforce Needs for the National Vision for Space Exploration, "Issues Affecting the Future of the U.S. Space Science and Engineering Workforce: Interim report", Technical report, National Academies, 2006.

[2] R. Stephens, "Ensuring Aerospace Skills of the Future: The birth to work pipeline", Technical Proceeding of AIAA/ICAS International Air and Space Symposium and Exposition: The Next 100 Years, Dayton, $\mathrm{OH}$, August 2003.

[3] D.H. Rhodes and D. Hastings, "The Case for Evolving Systems Engineering as a Field within Engineering Systems," MIT Engineering Systems Symposium, March 2004.

[4] H. Davidz, D. Nightingale, and D.Rhodes, "Enablers and Barriers to Systems Thinking Development: Results of a Qualitative and Quantitative Study," 3rd Conference on Systems Engineering Research, Hoboken, NJ, March 2005.

[5] D.Utter, Collaborative Distributed Systems Engineering, Master of Science Thesis, Engineering Systems Division, MIT, January 2007.

[6] C.T. Lamb, and D.H. Rhodes, "Standardized Process as a Tool for Higher Level Systems Thinking," INCOSE International Symposium 2007, San Diego, CA, June 2007.

[7] C.T. Lamb, D.H. Rhodes, and D.J. Nightingale, "Evolving Systems Engineering for the Realization of Effective Enterprise", $6^{\text {th }}$ Annual Conference on Systems Engineering Research, Los Angeles, CA, April 2008

[8] B. Glaser and A. Strauss, The Discovery of Grounded Theory. Aldine Publishing Company, Chicago, 1967.

[9] A. Strauss and J. Corbin, Basics of Qualitative Research. Sage Publications, Thousand Oaks, 1998.

[10] R. Yin, Case Study Research Design and Methods, Sage Publications, Thousand Oaks, 2003.

[11] R. Valerdi and H. Davidz, "Empirical Research in Systems Engineering: Challenges and opportunities of a new frontier", Technical Proceeding of 5th CSER Conference on Systems Engineering Research, Hoboken, NJ, March 2007.

[12] W. Rouse, Enterprises as systems: Essential challenges and enterprise transformation, Systems Eng 8(2)(2005), 138-150.

[13] A. Hall, A Methodology for Systems Engineering, NJ; Van Nostrand, 1962

[14] J. Moses, "ESD Monograph Framing Paper: Foundational Issues in Engineering Systems", Engineering Systems Monograph, MIT Engineering Systems Symposium, March 2004, Cambridge, MA

[15] D. H. Rhodes, New Imperatives for Educating Systems Engineering Leaders, INCOSE Insight, 2005

[16] P. Checkland, Systems Thinking, Systems Practice, Soft Systems Methodology: A 30-year retrospective, John Wiley and Sons, West Sussex, England, 1999.

[17] M. Frank, "Engineering Systems Thinking and Systems Thinking." Systems Engineering 3(3): 163-168, 2000

[18] M. Frank, "What is "Engineering Systems Thinking"?" Kybernetes 31(9/10): 1350-1360, 2002

[19] H. Davidz, Enabling Systems Thinking to Accelerate the Development of Senior Systems Engineers, PhD thesis, Massachusetts Institute of Technology, Cambridge, Massachusetts, 2006.

[20] D. H. Rhodes and D. Adams, "Report of Engineering Systems Thinking Research Study in a Government Agency", SEAri Working Paper, WP2007-7-1, 2007, http://seari.mit.edu

[21] A.M Ross and D.H. Rhodes, "Using Natural Value-Centric Time Scales for Conceptualizing System Timelines through Epoch-Era Analysis" SEAri Working Paper, WP-2007-1-3, 2007, http://seari.mit.edu

[22] C.T. Lamb and D.H. Rhodes, "Promoting Systems Thinking Through Alignment of Culture and Process: Initial Results," 5th Conference on Systems Engineering Research, Hoboken, NJ, March 2007. 